\title{
On finite groups with automorphisms whose fixed points are Engel
}

\author{
Pavel Shumyatsky and Danilo Sanção da Silveira \\ For Evgeny Khukhro on his 60th birthday
}

\begin{abstract}
The main result of the paper is the following theorem. Let $q$ be a prime, $n$ a positive integer and $A$ an elementary abelian group of order $q^{2}$. Suppose that $A$ acts coprimely on a finite group $G$ and assume that for each $a \in A^{\#}$ every element of $C_{G}(a)$ is $n$ Engel in $G$. Then the group $G$ is $k$-Engel for some $\{n, q\}$-bounded number $k$.
\end{abstract}

\section{Introduction}

Let $A$ be a finite group acting on a finite group $G$. Many wellknown results show that the structure of the centralizer $C_{G}(A)$ (the fixed-point subgroup) of $A$ has influence over the structure of $G$. The influence is especially strong if $(|A|,|G|)=1$, that is, the action of $A$ on $G$ is coprime. We will give examples illustrating this phenomenon.

Thompson proved that if $A$ is of prime order and $C_{G}(A)=1$, then $G$ is nilpotent [15. Higman proved that any nilpotent group admitting a fixed-point-free automorphism of prime order $q$ has nilpotency class bounded by some function $h(q)$ depending on $q$ alone [4]. The reader can find in [7] and [8] an account on the more recent developments related to these results. The next result is a consequence of the classification of finite simple groups [18]: If $A$ is a group of automorphisms of $G$ whose order is coprime to that of $G$ and $C_{G}(A)$ is nilpotent or has odd order, then $G$ is soluble. Once the group $G$ is known to be soluble, there is a wealth of results bounding the Fitting height of $G$ in

1991 Mathematics Subject Classification. 20D45, 20F40, $20 \mathrm{~F} 45$.

Key words and phrases. Finite groups, Automorphisms, Centralizers, Engel elements.

This work was supported by CNPq and FAPDF, Brazil. 
terms of the order of $A$ and the Fitting height of $C_{G}(A)$. This direction of research was started by Thompson in [16. The proofs mostly use representation theory in the spirit of the Hall-Higman work 3. A general discussion of these methods and their use in numerous fixed-point theorems can be found in Turull [17.

Following the solution of the restricted Burnside problem it was discovered that the exponent of $C_{G}(A)$ may have strong impact over the exponent of $G$. Remind that a group $G$ is said to have exponent $n$ if $x^{n}=1$ for every $x \in G$ and $n$ is the minimal positive integer with this property. The next theorem was obtained in $[\mathbf{9}$.

TheOREm 1.1. Let $q$ be a prime, $n$ a positive integer and $A$ an elementary abelian group of order $q^{2}$. Suppose that $A$ acts coprimely on a finite group $G$ and assume that $C_{G}(a)$ has exponent dividing $n$ for each $a \in A^{\#}$. Then the exponent of $G$ is $\{n, q\}$-bounded.

Here and throughout the paper $A^{\#}$ denotes the set of nontrivial elements of $A$. The proof of the above result involves a number of deep ideas. In particular, Zelmanov's techniques that led to the solution of the restricted Burnside problem [19, 20] are combined with the Lubotzky-Mann theory of powerful p-groups [12], Lazard's criterion for a pro- $p$ group to be $p$-adic analytic [10], and a theorem of Bakhturin and Zaicev on Lie algebras admitting a group of automorphisms whose fixed-point subalgebra is PI [1].

Let $n$ be a positive integer and let $x, y$ be elements of a group $G$. The commutators $\left[x_{n} y\right]$ are defined inductively by the rule

$$
\left[x,_{0} y\right]=x, \quad\left[x_{n} y\right]=\left[\left[x,_{n-1} y\right], y\right] .
$$

An element $x$ is called a (left) $n$-Engel element if for any $g \in G$ we have $\left[g{ }_{n} x\right]=1$. A group $G$ is called $n$-Engel if all elements of $G$ are $n$-Engel. The main result of the present article is the following theorem.

TheOREm 1.2. Let $q$ be a prime, $n$ a positive integer and $A$ an elementary abelian group of order $q^{2}$. Suppose that $A$ acts coprimely on a finite group $G$ and assume that for each $a \in A^{\#}$ every element of $C_{G}(a)$ is n-Engel in $G$. Then the group $G$ is $k$-Engel for some $\{n, q\}$ bounded number $k$.

The methods employed in the proof of Theorem 1.2 are roughly the same as those in the proof of Theorem 1.1. There are however some rather significant differences. The main difficulty is that Theorem 1.2 does not reduce as quickly as Theorem 1.1 to questions about Lie algebras over field. Instead, we have to work with Lie rings. This hurdle 
is overcome via Proposition 2.6 obtained in the next section and some other tools.

Throughout the paper we use without special references the wellknown properties of coprime actions:

If $\alpha$ is an automorphism of a finite group $G$ of coprime order, $(|\alpha|,|G|)=1$, then $C_{G / N}(\alpha)=C_{G}(\alpha) N / N$ for any $\alpha$-invariant normal subgroup $N$.

If $A$ is a noncyclic abelian group acting coprimely on a finite group $G$, then $G$ is generated by the subgroups $C_{G}(B)$, where $A / B$ is cyclic.

We use the expression " $\{a, b, \ldots\}$-bounded" to abbreviate "bounded from above in terms of $a, b, \ldots$ only".

\section{About Lie Rings and Lie Algebras}

Let $X$ be a subset of a Lie algebra $L$. By a commutator in elements of $X$ we mean any element of $L$ that can be obtained as a Lie product of elements of $X$ with some system of brackets. If $x_{1}, \ldots, x_{k}, x, y$ are elements of $L$, we define inductively

$$
\left[x_{1}\right]=x_{1} ;\left[x_{1}, \ldots, x_{k}\right]=\left[\left[x_{1}, \ldots, x_{k-1}\right], x_{k}\right]
$$

and $\left[x,_{0} y\right]=x ;\left[x,_{m} y\right]=\left[\left[x,_{m-1} y\right], y\right]$, for all positve integers $k, m$. As usual, we say that an element $a \in L$ is ad-nilpotent if there exists a positive integer $n$ such that $\left[x_{,_{n}} a\right]=0$ for all $x \in L$. If $n$ is the least integer with the above property then we say that $a$ is ad-nilpotent of index $n$.

The next theorem is a deep result of Zelmanov [20].

THEOREM 2.1. Let $L$ be a Lie algebra over a field and suppose that $L$ satisfies a polynomial identity. If $L$ can be generated by a finite set $X$ such that every commutator in elements of $X$ is ad-nilpotent, then $L$ is nilpotent.

An important criterion for a Lie algebra to satisfy a polynomial identity is provided by the next theorem. It was proved by Bakhturin and Zaicev for soluble groups $A$ [1] and later extended by Linchenko to the general case [11].

TheOREM 2.2. Let $L$ be a Lie algebra over a field $K$. Assume that a finite group $A$ acts on $L$ by automorphisms in such a manner that $C_{L}(A)$ satisfies a polynomial identity. Assume further that the characteristic of $K$ is either 0 or prime to the order of $A$. Then $L$ satisfies a polynomial identity.

Both Theorem 2.1 and Theorem 2.2 admit respective quantitative versions (see for example [14]). 
THEOREM 2.3. Let $L$ be a Lie algebra over a field $K$ generated by $a_{1}, \ldots, a_{m}$. Suppose that $L$ satisfies a polynomial identity $f \equiv 0$ and each commutator in $a_{1}, \ldots, a_{m}$ is ad-nilpotent of index at most $n$. Then $L$ is nilpotent of $\{f, K, m, n\}$-bounded class.

TheOREm 2.4. Let $L$ be as in Theorem 2.2 and assume that $C_{L}(A)$ satisfies a polynomial identity $f \equiv 0$. Then $L$ satisfies a polynomial identity of $\{|A|, f, K\}$-bounded degree.

By combining the above results we obtain the following corollary.

Corollary 2.5. Let $L$ be a Lie algebra over a field $K$ and $A$ a finite group of automorphisms of $L$ such that $C_{L}(A)$ satisfies the polynomial identity $f \equiv 0$. Suppose that the characterisitic of $K$ is either 0 or prime to the order of $A$. Assume that $L$ is generated by an $A$-invariant set of $m$ elements in which every commutator is ad-nilpotent of index at most $n$. Then $L$ is nilpotent of $\{|A|, f, K, m, n\}$-bounded class.

We will need a similar result for Lie rings. As usual, $\gamma_{i}(L)$ denotes the $i$ th term of the lower central series of $L$.

Proposition 2.6. Let $L$ be a Lie ring and $A$ a finite group of automorphisms of $L$ such that $C_{L}(A)$ satisfies the polynomial identity $f \equiv 0$. Further, assume that $L$ is generated by an $A$-invariant set of $m$ elements such that every commutator in the generators is ad-nilpotent of index at most $n$. Then there exist positive integers e and c, depending only on $|A|, f, m$ and $n$, such that $e \gamma_{c}(L)=0$.

Proof. Since the set of generators of $L$ is $A$-invariant, every automorphism $\alpha \in A$ induces a permutation of the generators. Let $L_{\mathbb{Q}}$ denote the free Lie algebra on free generators $x_{1}, \ldots, x_{m}$ over the field $\mathbb{Q}$ of rational numbers and $L_{\mathbb{Z}}$ the Lie subring of $L_{\mathbb{Q}}$ generated by $x_{1}, \ldots, x_{m}$. For each $\alpha \in A$ let $\phi_{\alpha}$ be the automorphism of $L_{\mathbb{Q}}$ (or of $L_{\mathbb{Z}}$ ) such that $x_{i}^{\phi_{\alpha}}=x_{\pi(i)}$ where $\pi$ is the permutation that $\alpha$ induces on the generators of $L$. The mapping that takes $\alpha \in A$ to $\phi_{\alpha}$ induces a natural action of $A$ on $L_{\mathbb{Q}}$ (or on $L_{\mathbb{Z}}$ ) by automorphisms.

Let $I$ be the ideal of $L_{\mathbb{Z}}$ generated by all values of $f$ on elements of $C_{L_{\mathbb{Z}}}(A)$ and by all elements of the form $\left[u,{ }_{n} v\right]$, where $u$ ranges through $L_{\mathbb{Z}}$ and $v$ ranges through the set of all commutators in the generators $x_{1}, \ldots, x_{m}$. By Corollary 2.5$] L_{\mathbb{Q}} / \mathbb{Q} I$ is nilpotent of $\{|A|, f, m, n\}$ bounded class, say $c-1$.

If $y_{1}, \ldots, y_{c}$ are not necessarily distinct elements of the set $x_{1}, \ldots, x_{m}$, it follows that the commutator $\left[y_{1}, \ldots, y_{c}\right]$ lies in $\mathbb{Q} I$ and therefore it 
can be written in the form

$$
\left[y_{1}, \ldots, y_{c}\right]=\sum_{i} q_{i} f_{i} w_{i}
$$

where $q_{i}$ are rational numbers, $f_{i}$ are either elements of the form $\left[u,{ }_{n} v\right]$ as above or values of $f$ in elements of $C_{L_{\mathbb{Z}}}(A)$ and $f_{i} w_{i}$ are elements of $L_{\mathbb{Z}}$ obtained by multiplying (several times) $f_{i}$ with products in the free generators $x_{1}, \ldots, x_{m}$.

Let $e$ be the least common multiple of the denominators of the coefficients $q_{i}$ taken over all possible choices of $y_{1}, \ldots, y_{c}$ in $\left\{x_{1}, \ldots, x_{m}\right\}$. Then $e\left[y_{1}, \ldots, y_{c}\right] \in I$ for any choice of $y_{1}, \ldots, y_{c}$. Therefore $e \gamma_{c}\left(L_{\mathbb{Z}}\right) \leq$ $I$.

We remark that there exists a natural homomorphism of $L_{\mathbb{Z}} / I$ onto $L$ under which each automorphism $\phi_{\alpha}$ induces the automorphism $\alpha$ of $L$. Thus, $e \gamma_{c}(L)=0$, as required.

We will now quote a useful lemma from [9].

LEMMA 2.7. Let $L$ be a Lie ring and $H$ a subring of $L$ generated by $m$ elements $h_{1}, \ldots, h_{m}$ such that all commutators in $h_{i}$ are ad-nilpotent in $L$ of index at most $n$. If $H$ is nilpotent of class $c$, then for some $\{c, m, n\}$-bounded number $u$ we have $[L, \underbrace{H, \ldots, H}_{u}]=0$.

Recall that the identity

$$
\sum_{\sigma \in S_{n}}\left[y, x_{\sigma(1)}, \ldots, x_{\sigma(n)}\right] \equiv 0
$$

is called linearized $n$-Engel identity. In general, Theorem 2.1 cannot be extended to the case where $L$ is just a Lie ring (rather than Lie algebra over a field). However such an extension does hold in the particular case where the polynomial identity $f \equiv 0$ is a linearized Engel identity.

THEOREM 2.8. Let $f$ be a Lie polynomial of degree $n$ all of whose coefficients equal 1 or -1 . Let $L$ be a Lie ring generated by finitely many elements $a_{1}, \ldots, a_{m}$ such that all commutators in the generators are ad-nilpotent of index at most $n$. Assume that $L$ satisfies the identity $f \equiv 0$. Then $L$ is nilpotent of $\{f, m, n\}$-bounded class.

The deduction of Theorem 2.8 from Theorem 2.3 uses just standard arguments. For the reader's convenience we sketch out a proof.

Proof. By Proposition 2.6 applied with $A=1$, there exist positive integers $e$ and $c$, depending only on $f, m$ and $n$, such that $e \gamma_{c}(L)=0$. We set $M=e L$ and notice that $M$ is a nilpotent ideal of $L$ whose nilpotency class is bounded in terms of $f, m$ and $n$. Let $d$ be the 
derived length of $M$ and use induction on $d$. Suppose first that $M=$ 0 . Then $L$ is a direct sum of its primary components $L_{p}$, where $p$ ranges through prime divisors of $e$. It is sufficient to show that the nilpotency class of each $L_{p}$ is bounded in terms of $f, m$ and $n$ and so without loss of generality we can assume that $e=p^{k}$ for a prime $p$. We note that $k$ here is $\{f, m, n\}$-bounded. The quotient $L / p L$ can be regarded as a Lie algebra over the field with $p$ elements and so it is nilpotent of bounded class by Theorem 2.3 . Hence, there exists an $\{f, m, n\}$-bounded number $c_{1}$ such that $\gamma_{c_{1}}(L) \leq p L$. This implies that $\gamma_{k c_{1}}(L) \leq p^{k} L=0$ and we are done.

Assume now that $M \neq 0$ and let $D$ be the last nontrivial term of the derived series of $M$. By induction $\bar{L}=L / D$ is nilpotent of bounded class. The ring $\bar{L}$ naturally acts on $D$ by derivations and Lemma 2.7 shows that for some $\{f, m, n\}$-bounded number $u$ we have $[D, \underbrace{\bar{L}, \ldots, \bar{L}}_{u}]=0$. It follows that $L$ is nilpotent of $\{f, m, n\}$-bounded class, as required.

\section{On associated Lie rings}

There are several well-known ways to associate a Lie ring to a group (see [6, 7, 14]). For the reader's convenience we will briefly describe the construction that we are using in the present paper.

Let $G$ be a group. A series of subgroups

$$
G=G_{1} \geq G_{2} \geq \ldots
$$

is called an $N$-series if it satisfies $\left[G_{i}, G_{j}\right] \leq G_{i+j}$ for all $i, j$. Obviously any $N$-series is central, i.e. $G_{i} / G_{i+1} \leq Z\left(G / G_{i+1}\right)$ for any $i$. Given an $N$-series $(*)$, let $L^{*}(G)$ be the direct sum of the abelian groups $L_{i}^{*}=G_{i} / G_{i+1}$, written additively. Commutation in $G$ induces a binary operation [,] in $L^{*}(G)$. For homogeneous elements $x G_{i+1} \in L_{i}^{*}, y G_{j+1} \in$ $L_{j}^{*}$ the operation is defined by

$$
\left[x G_{i+1}, y G_{j+1}\right]=[x, y] G_{i+j+1} \in L_{i+j}^{*}
$$

and extended to arbitrary elements of $L^{*}(G)$ by linearity. It is easy to check that the operation is well-defined and that $L^{*}(G)$ with the operations + and [,] is a Lie ring. If all quotients $G_{i} / G_{i+1}$ of an $N$ series $(*)$ have prime exponent $p$ then $L^{*}(G)$ can be viewed as a Lie algebra over the field with $p$ elements. Any automorphism of $G$ in the natural way induces an automorphism of $L^{*}(G)$. If $G$ is finite and $\alpha$ is an automorphism of $G$ such that $(|\alpha|,|G|)=1$, then the subring of fixed points of $\alpha$ in $L^{*}(G)$ is isomorphic with the Lie ring associated to 
the group $C_{G}(\alpha)$ via the series formed by intersections of $C_{G}(\alpha)$ with the series $(*)$.

In the case where the series $(*)$ is just the lower central series of $G$ we write $L(G)$ for the associated Lie ring. In the case where the series $(*)$ is the $p$-dimension central series of $G$ we write $L_{p}(G)$ for the subalgebra generated by the first homogeneous component $G_{1} / G_{2}$ in the associated Lie algebra over the field with $p$ elements.

Proposition 3.1. Let $G$ be an m-generated group satisfying the hypothesis of Theorem 1.2. Let $p$ be a prime number that divides the order of $G$. Then

(1) $L_{p}(G)$ is nilpotent of $\{p, q, m, n\}$-bounded class.

(2) There exists positive integers e, $c$ depending only on $m, n$ and $q$, such that $e \gamma_{c}(L(G))=0$.

The proofs of the statements (1) and (2) are similar. We will give a detailed proof of the second statement only. The proof of (1) can be obtained simply by replacing every appeal to Proposition 2.6 in the proof of (2) by an appeal to Corollary 2.5. An important observation that is used in the proof is that if $G$ is a finite $p$-group that can be generated by $d$ elements, then we can choose $d$ generators from any subset that generates $G$. This follows from the well-known Burnside Basis Theorem [5].

Proof. Since $G$ is generated by the centralizers $C_{G}(a)$, where $a \in$ $A^{\#}$, and since all elements in the centralizers $C_{G}(a)$ are Engel, the Baer Theorem [[5], III,6.14] implies that the group $G$ is nilpotent. Therefore $G$ is a direct product of its Sylow subgroups. Hence, without loss of generality we can assume that $G$ is a $p$-group. Let $\gamma_{j}=\gamma_{j}(G)$, $L_{j}=\gamma_{j} / \gamma_{j+1}$ and $L=L(G)=\oplus_{j \geq 1} L_{j}$ be the Lie ring associated with the group $G$. Let $A_{1}, \ldots, A_{q+1}$ be the distinct maximal subgroups of $A$. Set $L_{i j}=C_{L_{j}}\left(A_{i}\right)$. We know that any $A$-invariant subgroup is generated by the centralizers of $A_{i}$. Therefore for any $j$ we have

$$
L_{j}=\sum_{i=1}^{q+1} L_{i j} .
$$

Further, for any $l \in L_{i j}$ there exists $x \in \gamma_{j} \cap C_{G}\left(A_{i}\right)$ such that $l=x \gamma_{j+1}$. Since $x$ is $n$-Engel in $G$, it follows that $l$ is ad-nilpotent of index at most $n$. Thus,

any element in $L_{i j}$ is ad-nilpotent in $L$ of index at most $n$. 
Since $G$ is generated by $m$ elements, the additive group $L_{1}$ is generated by $m$ elements. It follows that the Lie ring $L$ is generated by at most $m$ ad-nilpotent elements, each from $L_{i 1}$ for some $i$.

Let $\omega$ be a primitive $q$ th root of unity and consider the tensor product $\bar{L}=L \otimes \mathbb{Z}[\omega]$. Set $\bar{L}_{j}=L_{j} \otimes \mathbb{Z}[\omega]$ for $j=1,2, \ldots$ We regard $\bar{L}$ as a Lie ring and so $\bar{L}=\left\langle\bar{L}_{1}\right\rangle$. Since the additive subgroup $L_{1}$ is generated by $m$ elements, it follows that the additive subgroup $\bar{L}_{1}$ is generated by $(q-1) m$ elements. We also remark that there is a natural embedding of the ring $L$ into the ring $\bar{L}$.

The group $A$ acts on $\bar{L}$ in the natural way and we have $\bar{L}_{i j}=$ $C_{\bar{L}_{j}}\left(A_{i}\right)$ where $\bar{L}_{i j}=L_{i j} \otimes \mathbb{Z}[\omega]$. We will now establish the following claim.

Any element in $\bar{L}_{i j}$ is ad-nilpotent in $\bar{L}$ with $\{n, q\}$-bounded index.

Indeed choose $y \in \bar{L}_{i j}$ and write

$$
y=x_{0}+\omega x_{1}+\omega^{2} x_{2}+\cdots+\omega^{q-2} x_{q-2}
$$

for suitable $x_{s} \in L_{i j}$. In view of (11) it is easy to see that each of the summands $\omega^{s} x_{s}$ is ad-nilpotent in $\bar{L}$ of index at most $n$.

Let $H=\left\langle x_{0}, \omega x_{1}, \omega^{2} x_{2}, \ldots, \omega^{q-2} x_{q-2}\right\rangle$ be the subring of $\bar{L}$ generated by $x_{0}, \omega x_{1}, \omega^{2} x_{2}, \ldots, \omega^{q-2} x_{q-2}$. We wish to show that $H$ is nilpotent of $\{n, q\}$-bounded class.

Note that $H \leq C_{\bar{L}}\left(A_{i}\right)$ since $\omega^{s} x_{s} \in C_{\bar{L}}\left(A_{i}\right)$ for all $s$. A commutator of weight $t$ in the elements $x_{0}, \omega x_{1}, \omega^{2} x_{2}, \ldots, \omega^{q-2} x_{q-2}$ has the form $\omega^{t} x$, for some $x \in L_{i j_{0}}$, where $j_{0}=t j$. By (1), the element $x$ is ad-nilpotent of index at most $n$ in $L$ and so we deduce that $\omega^{t} x$ is ad-nilpotent of index at most $n$ in $\bar{L}$.

Since $C_{G}\left(A_{i}\right)$ is $n$-Engel, we conclude that $C_{L}\left(A_{i}\right)$ satisfies the linearized $n$-Engel identity. This identity is multilinear and so it is also satisfied by $C_{L}\left(A_{i}\right) \otimes \mathbb{Z}[\omega]=C_{\bar{L}}\left(A_{i}\right)$. Since $H \leq C_{\bar{L}}\left(A_{i}\right)$, it follows that the identity is satisfied by $H$. Hence, by Theorem 2.8 , $H$ is nilpotent of $\{n, q\}$-bounded class.

By Lemma 2.7, there exists a positive integer $v$, depending only on $n$ and $q$, such that $\left[\bar{L}_{v} H\right]=0$. Since $y \in H$, we conclude that $y$ is ad-nilpotent in $\bar{L}$ with $\{n, q\}$-bounded index. This proves Claim (2).

An element $x \in \bar{L}$ will be called a common "eigenvector" for $A$ if for any $a \in A$ there exists $s$ such that $x^{a}=\omega^{s} x$. Since $(|A|,|G|)=1$, the additive group of the Lie ring $\bar{L}$ is generated by common eigenvectors for $A$ (see for example [7, Lemma 4.1.1]). 
We have already remarked that the additive subgroup $\bar{L}_{1}$ is generated by $(q-1) m$ elements. It follows that the Lie ring $\bar{L}$ is generated by at most $(q-1) m$ common eigenvectors for $A$. Certainly, the Lie ring $\bar{L}$ is generated by an $A$-invariant set of at most $(q-1) q m$ common eigenvectors for $A$.

Since $A$ is noncyclic, any common eingenvector for $A$ is contained in the centralizer $C_{\bar{L}}\left(A_{i}\right)$ for some $i$. Further, any commutator in common eigenvectors is again a common eigenvector. Therefore if $l_{1}, \ldots, l_{s} \in \bar{L}_{1}$ are common eigenvectors for $A$ generating $\bar{L}$, then any commutator in these generators belongs to some $\bar{L}_{i j}$ and therefore, by Claim (2), is ad-nilpotent of $\{n, q\}$-bounded index.

As we have mentioned earlier, for any $i$ the subalgebra $C_{\bar{L}}\left(A_{i}\right)$ satisfies the linearized $n$-Engel identity. Thus, by Proposition 2.6, there exist positive integers $e, c$ depending only on $m, n$ and $q$ such that $e \gamma_{c}(\bar{L})=0$. Since $L$ embeds into $\bar{L}$, we also have $e \gamma_{c}(L)=0$. The proof is complete.

\section{Proof of the main theorem}

A well-known theorem of Gruenberg says that a soluble group generated by finitely many Engel elements is nilpotent (see [13. 12.3.3]). We will require a quantitative version of this theorem.

LEMMA 4.1. Let $G$ be a group generated by $m$ n-Engel elements and suppose that $G$ is soluble with derived length $d$. Then $G$ is nilpotent of $\{d, m, n\}$-bounded class.

Proof. Suppose that the lemma is false. Then for each positive integer $i$ we can choose a group $G_{i}$ satisfying the hypothesis of the lemma and having nilpotency class at least $i$. In each group $G_{i}$ we fix generators $g_{i 1}, g_{i 2}, \ldots, g_{i m}$ that are $n$-Engel elements.

In the Cartesian product of the groups $G_{i}$ consider the subgroup $D$ generated by $m$ elements

$$
g_{1}=\left(g_{11}, g_{21}, \ldots\right), \ldots, g_{m}=\left(g_{1 m}, g_{2 m} \ldots\right) .
$$

Thus, $D$ is a soluble group generated by $m$ elements that are $n$-Engel. By Gruenberg's theorem $D$ is nilpotent, say of class $c$. We remark that each of the groups $G_{i}$ is isomorphic to a quotient $D$. Hence each of the groups $G_{i}$ is nilpotent of class at most $c$. A contradiction.

Given a group $H$ and an integer $i$, in what follows we write $H^{i}$ for the subgroup of $H$ generated by all $i$ th powers and $\gamma_{i}(H)$ for the $i$ th term of the lower central series of $H$. We will also require the concept of powerful $p$-groups. These were introduced by Lubotzky and Mann 
in [12]: a finite $p$-group $G$ is powerful if and only if $G^{p} \geq[G, G]$ for $p \neq 2$ (or $G^{4} \geq[G, G]$ for $p=2$ ). The reader can consult books [2] or 8 for more information on these groups.

Powerful $p$-groups have many nice properties. In particular, if $P$ is a powerful $p$-group, the subgroups $\gamma_{i}(P)$ and $P^{i}$ are also powerful. Moreover, if $n_{1}, \ldots, n_{s}$ are positive integers, it follows by repeated applications of [12, Props. 1.6 and 4.1.6] that

$$
\left[P^{n_{1}}, \ldots, P^{n_{s}}\right] \leq \gamma_{s}(P)^{n_{1} \cdots n_{s}} .
$$

If $P$ is generated by $d$ elements, then any subgroup of $P$ can be generated by at most $d$ elements and $P$ is a product of $d$ cyclic subgroups (see Theorem 1.11 and Theorem 1.12 in [12]).

We are now ready to prove Theorem 1.2 .

Proof of Theorem 1.2. Let $G$ be as in Theorem 1.2, We wish to show that $G$ is $k$-Engel for some $\{n, q\}$-bounded number $k$. Since $G$ is generated by the centralizers $C_{G}(a)$ with $a \in A^{\#}$ and since all elements in the centralizers $C_{G}(a)$ are Engel, the Baer Theorem implies that the group $G$ is nilpotent. Choose arbitrarily $x, y \in G$. It is sufficient to show that the subgroup $\langle x, y\rangle$ is nilpotent of $\{n, q\}$-bounded class. Without loss of generality we can assume that no proper $A$ invariant subgroup of $G$ contains both $x$ and $y$. In other words, we will assume that $G=\left\langle x^{A}, y^{A}\right\rangle$. Therefore the group $G$ can be generated by $2 q^{2}$ elements. Every Sylow subgroup of $G$ satisfies these hypotheses and therefore without loss of generality we can assume that $G$ is a $p$-group for some prime $p \neq q$. Using the fact that $G$ is generated by the centralizers $C_{G}(a)$ with $a \in A^{\#}$ and combining it with the Burnside Basis Theorem we can assume further that $G$ is generated by at most $2 q^{2}$ elements which are $n$-Engel. In view of Lemma 4.1 it is now sufficient to show that the derived length of $G$ is $\{n, q\}$-bounded. Let $L=L(G)$. By Proposition 3.1 (2), there exist positive integers $e, c$ that depend only on $n$ and $q$ such that $e \gamma_{c}(L)=0$. If $p$ is not a divisor of $e$, we have $\gamma_{c}(L)=0$ and so the group $G$ is nilpotent of class at most $c-1$. In that case the proof is complete and so we assume that $p$ is a divisor of $e$. Consider first the particular case where $G$ is a powerful $p$-group. Set $R=G^{e}$ and assume that $R \neq 1$.

We have

$$
[R, R] \leq[G, G]^{e^{2}} \leq G^{p e^{2}}=R^{p e}
$$

Let $L_{1}=L(R)$. By Proposition $3.1(2), e \gamma_{c}\left(L_{1}\right)=0$. The fact that $e \gamma_{c}\left(L_{1}\right)=0$ means that $\gamma_{c}(R)^{e} \leq \gamma_{c+1}(R)$. Taking into account that $R$ is powerful, we write

$$
\gamma_{c}(R)^{e} \leq \gamma_{c+1}(R)=\left[R^{\prime}{ }_{c-1} R\right] \leq\left[R^{p e}{ }_{c-1} R\right] \leq \gamma_{c}(R)^{p e} .
$$


Hence, $\gamma_{c}(R)^{e}=1$. Since $\gamma_{c}(R)$ is powerful and generated by at most $2 q^{2}$ elements, we conclude that $\gamma_{c}(R)$ is a product of at most $2 q^{2}$ cyclic subgroups. Hence the order of $\gamma_{c}(R)$ is at most $e^{2 q^{2}}$. It follows that the derived length of $R$ is $\{n, q\}$-bounded. Recall that $G$ is a powerful $p$-group and $R=G^{e}$. It follows that the derived length of $G$ is $\{n, q\}$ bounded. Thus, we proved the desired result in the case where $G$ is a powerful $p$-group.

Let us now drop the assumption that $G$ is powerful. By Proposition 3.1 (1) the algebra $L_{p}(G)$ is nilpotent with bounded nilpotency class. Proposition 1 of $[9$ now tells us that $G$ has a characteristic powerful subgroup of bounded index. We already know that the derived length of the powerful subgroup is $\{n, q\}$-bounded. Hence, the derived length of $G$ is $\{n, q\}$-bounded as well. The proof is now complete.

\section{References}

[1] Y. A. Bakhturin, M. V. Zaicev, Identities of graded algebras, J. Algebra, 205 (1998), 1-12.

[2] J.D. Dixon, M.P.F. du Sautoy, A. Mann, and D. Segal, Analytic Pro-p Groups, Cambridge University Press, Cambridge, 1991.

[3] P. Hall, G. Higman, The $p$-length of a $p$-soluble group and reduction theorems for Burnside's problem, Proc. London Math. Soc., (3) 6 (1956), 1-42.

[4] G. Higman, Groups and Lie rings having automorphisms without non-trivial fixed points, J. London Math. Soc., 32 (1957), 321-334.

[5] B. Huppert, Endliche Gruppen I, Springer-Verlag, 1967.

[6] B. Huppert, N. Blackburn, Finite groups II. Springer Verlang, Berlin, 1982.

[7] E.I. Khukhro, Nilpotent Groups and their Automorphisms, de Gruyter-Verlag, Berlin, 1993.

[8] E.I. Khukhro, p-Automorphisms of Finite p-Groups, London Math. Soc. Lecture Note Series 246, Cambridge University Press, Cambridge, 1998.

[9] E.I. Khukhro, P. Shumyatsky, Bounding the exponent of a finite group with automorphisms, J. Algebra, 212 (1999), 363-374.

[10] M. Lazard, Groupes Analytiques p-Adiques, Publ. Math. Inst. Hautes Études Sci., 26, Paris, (1965), 389-603.

[11] V. Linchenko, Identities of Lie algebras with actions of Hopf algebras, Comm. Algebra, 25 (1997), 3179-3187.

[12] A. Lubotzky, A. Mann, Powerful p-groups. I: finite groups, J. Algebra, 105 (1987), 484-505.

[13] D.J.S. Robinson, A course in the theory of groups, 2nd ed. Springer-Verlag, New York, 1996.

[14] P. Shumyatsky, Applications of Lie ring methods to group theory, in Nonassociative Algebra and Its Applications, (Eds R. Costa et al.), Marcel Dekker, New York, (2000), 373-395. 
[15] J.G. Thompson, Finite groups with fixed-point-free automorphisms of prime order, Proc. Nat. Acad. Sci. USA, 45 (1959), 578-581.

[16] J.G. Thompson, Automorphisms of solvable groups, J. Algebra, 1 (1964), 259267.

[17] A. Turull, Character theory and length problems, in Finite and Locally Finite Groups, Kluwer Academic Publ., NATO ASI Series 471, Dordrecht-BostonLondon, 1995, 377-400.

[18] Y.M. Wang, Z.M. Chen, Solubility of finite groups admitting a coprime order operator group, Boll. Un. Mat. Ital. A, 7 (1993), 325-331.

[19] E. Zelmanov, Lie methods in the theory of nilpotent groups, in Groups '93 Galaway/ St Andrews, Cambridge University Press, Cambridge, (1995), 567585.

[20] E. Zelmanov, Nil Rings and Periodic Groups, The Korean Math. Soc. Lecture Notes in Math., Seoul, (1992).

Department of Mathematics, University of Brasilia, 70910-900 Brasília DF, BRAZIL

E-mail address: pavel@unb.br

Department of Mathematics, University of Brasilia, 70910-900 Brasília DF, BRAZIL

E-mail address: sancaodanilo@ufg.br 